\title{
Modeling on microcomputers
}

\author{
C. MICHAEL LEVY and MARC W. DURNIN \\ University of Florida, Gainesville, Florida 32611
}

\begin{abstract}
The instructional power of experimental models was recognized by the significant effort directed toward the development of EXPER SIM at several institutions. Owing to a number of factors, interest in these tools appeared to wane. The price/performance characteristics of some new microcomputers suggested that a rekindling of interest in the use of modeling as an inquiry/ problem solving tool could possibly be achieved if the large timesharing versions could be successfully migrated to one or more popular micros. We have partially achieved these objectives, having both transported EXPER SIM to the LSI-11 computer and developed, tested, documented, and evaluated many new models in sensory, personality, and social peychology and psychobiology. In addition, we have begun devising techniques to migrate a version to the Apple II and III microcomputers.
\end{abstract}

At the University of Florida the number of students who might enroll in a laboratory course usually makes infeasible the traditional "hands on" approach. We have, instead, developed these courses around a flexible system of computer simulations and models, while at the same time trying to preserve a classic value (Levy, 1980). Last year at these meetings White (1980) described how we relate the simulations and modeling experiences with limited "hands on" work in a sensation and perception laboratory course, and Fischler (1980) gave a related overview of the cognitive laboratory course. This presentation is more general, focusing upon the ways that modeling programs can be effectively used in a variety of classroom situations.

Nearly a decade ago, when Main (Main \& Head, 1971) and Stout (1974) began work on their innovative modeling system, EXPER SIM, they had only 1 degree of freedom to use in mounting it-the large University of Michigan computer. While incredibly sophisticated, the MESS system (as it became known) suffered by today's pedagogical standards because it was totally batch oriented. A version, known by the acronym LESS, was developed within a few years at the University of Louisville by Thurmond and Cromer (1975). LESS overcame the problems with batch operation, as did the adaptation by Spelt and Warden (1975) at Wabash College, known as WRIST. Still, because LESS and WRIST required a minimum of almost $\$ 70,000$ for hardware, operating system, and language licenses (or, at least, remote access to a medium-sized interactive installation), matters of money and geog-

Work on this project was supported by a CAUSE grant from the National Science Foundation (SER 78-06025) to the first author. The views and opinions expressed herein do not necessarily reflect those of the Foundation. Requests for reprints should be directed to C. Michael Levy, 114 Psychology Building, University of Florida, Gainesville, Florida 32611. raphy interacted adversely with instructional enthusiasm, yielding a sad bottom line: namely, comparatively few institutions interfaced EXPER SIM into their curricula. Another factor that negatively influenced widespread implementation ultimately emerged: There seemed to be no convenient forum, much less a network, to enable veteran and virginal users to communicate on a continuing basis about new models. Along with Eckerman (1981) and Spelt (1981), we are trying to rekindle some of the enthusiasm about modeling in the classroom that Dana Main years ago instilled in me.

What I want to convey here is an introduction for the newcomer to some of the virtues of these tools in the instructional environment. I also want to introduce more experienced users to the range of new models developed at the University of Florida under a new version of EXPER SIM, that go under the acronym FIRM (Florida InteRactive Modeler), which may facilitate off-loading courses from minicomputers and large mainframes to microcomputers. We will have solved the dissemination problem, in part, when CONDUIT begins distribution next year of the FIRM system and a core of models; CONDUIT's commitment to this project should offer encouragement to others who wish to add to the model base. An open forum, such as that planned for these meetings, undoubtedly will help.

The basic idea of an instructional model is very simple: A student particularizes an experimental environment and immediately observes the outcome. More specifically, a host of independent variables can be manipulated (or controlled or ignored), one or more dependent variables can be selected for measurement, and raw or transformed data for groups or individual subjects can be displayed. The possible manipulations are subject only to the ingenuity of the model developers and their knowledge of the field. (It should be noted, however, that developers sometimes must be 
willing to interpolate across wide gaps in the literature.) The effects, as revealed to the student, will show no more or less power and reliability than that revealed to the researchers who collected and reported the data upon which the model is built.

The experimental approach of the student can range from the commonplace, "What would happen if I used more subjects?" to an elaborate program of interrelated experiments. Novice students can be allowed to work with only a small subset of variables; advanced users can be given access to the whole gamut of options. Students may even examine the effects of variables that do not exist within the formal model; at the instructor's discretion, these unique independent variables can have either no effect at all or one of 100 different combinations of magnitude and functional relationship to a given behavior. Cost functions (including, for example, subject fees, stimulus preparation, and administrative overhead) can be activated and students given a "research grant" to spend as they please, quickly coming to the realization that careful planning of an endeavor is the most economical way of finding answers. The models can be written to yield data appropriate for a particular statistic being taught, giving the students a logical rationale for learning the new tool. Manipulations can be made both between and within subjects, enabling students to observe directly and form their own conclusions about the relative sensitivity of these designs in particular domains.

There are a host of other virtues associated with using models in the classroom that Main and Head (1971), Stout (1974), and Thurmond and Cromer (1975) have identified. For us, the most exciting promise of models is their capability to compress and expand real time. I am not referring here to the fact that sub. stantial hours are saved over traditional "hands on" research, although the models do, in fact, accomplish these ends through the elimination of the requirements for equipment set up, data collection, transcription, and so forth. What $I$ am trying to indicate is that it is possible with these models to study domains that would not be ordinarily feasible in real life. Our program in behavior genetics, for example, allows for the selection, breeding, and testing of thousands of generations of rodents in less time than one female rat can gestate. A model based upon the evoked potential, in contrast, could allow temporal stretching of the response to permit very fine-grained analyses.

We now have, in various stages of completion, 17 EXPER SIM models adapted or especially created for the FIRM system. (We do not consider a model complete until the data base, the student manual, and the instructor guides have been thoroughly field tested.) These models include: the cocktail-party simulation for cognitive psychology; imprinting for comparative psychology (both modified from their LESS and MESS versions); models focusing upon conformity, crowding, persuasion, aggression, attitudes, and the sleeper effect for courses in social and personality; units on dark adaptation and the McCollough effect for sensation and perception; and five new models aimed at students in psychobiology and two for students in developmental psychology.

From the student's view, the use of a model appears to be exactly the same as with LESS and WRIST. A portion of a sample dialogue appears in Table 1. Note that the student may or may not be prompted on the full labels for the variables (prompting takes considerable time on older Teletypes), that the system does considerable checking for the legality of input, and access to the hidden and secondary variables is functionally equivalent to that provided by WRIST and LESS. Note also that, like LESS and WRIST but unlike the MESS FORTRAN version, students must execute one cell at a time. Obviously, this takes more time per run than specifying a large complete factorial design at the outset, but CPU time charges are irrelevant on a dedicated machine. More importantly, the student must plan one step at a time, is encouraged to reflect upon the outcome of that run before attempting the next phase, and is not overwhelmed with pages of output. These factors make use of the system considerably easier for students and simplify teaching by the instructor.

From the author or model developer's perspective, the FIRM is substantially different from its predecessors. For one thing, the system is highly modularized in order to allow the individual components to fit within very limited memory. For another, a number of new features were added to facilitate the design and development, as well as the testing, debugging, and large-scale modification of models. A summary flowchart of the system is shown in Table 2. Models may be entered directly

Table 1

Sample Dialogue: Cocktail-Party Simulation

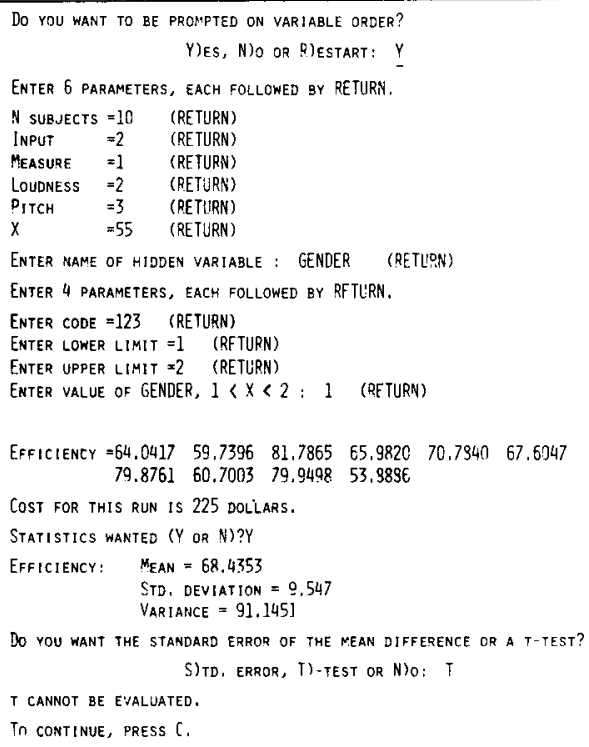


Table 2

Summary of FIRM

INSTRUCTOR SECTION

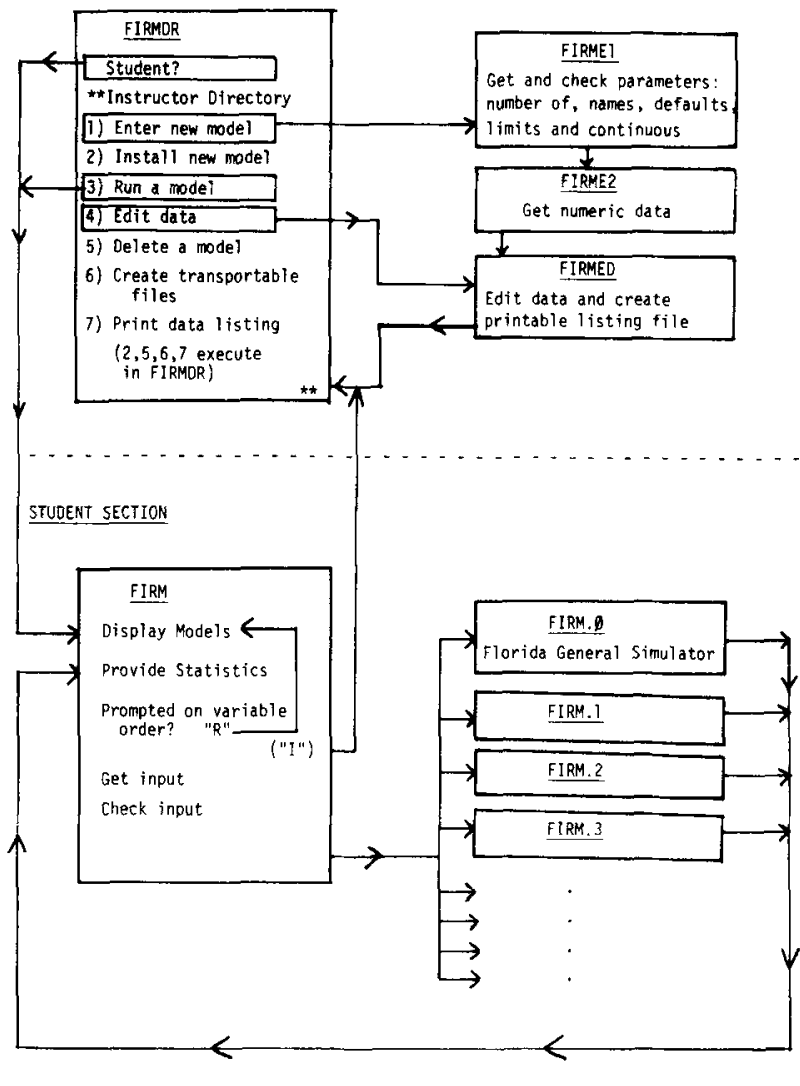

by the author as they are created, or models developed elsewhere can easily be installed on the system. Locally created or imported installed models can be tested in the run mode, modified in the edit mode, and may be interactively fine-tuned until the developer is satisfied. Models temporarily created during the early phases of an author's work at the terminal or perhaps by students as specific course assignments may be readily purged from the system. The system can create the needed numeric, string, and program files for installation at different sites. Recognizing that developers do not always perform all development and testing at an on-line terminal, the system provides a capability to print for any model a fully formatted and labeled listing of experiment and variable names plus values of numeric data (model parameters, defaults, limits, continuous variable information-if any-effects, and costs).

A major component of the system is the Florida General Simulator (FGS), an extremely powerful tool for interactively creating new models. The FGS prompts the novice developer through an extensive dialogue, at the completion of which the model is ready for debugging and running. Thus, anyone with the substantive knowledge of the process or phenomena addressed by a model, but with no programming skills, may create new models rather painlessly. One important proviso, however, needs to be mentioned about the FGS: The problem must be expressed in terms of a linear additive (e.g., analysis of variance) model. In the FGS each dependent variable is computed in the following manner: Each term in the equation is assigned a specific weight and a specific effect (or set of effects) with interactions between designated independent variables. These weightby-effect terms are summed, a constant amount (which could be zero) is added, and an error term (a proportion multiplied by a random number between -1 and +1 ) is algebraically added to the dependent measure.

Obviously, not all candidate problems are appropriate for the linear additive model required by the FGS. In fact, in the University of Florida undergraduate teaching laboratory, we have had no occasion to use the FGS. Creating new models from scratch (i.e., not using the FGS) will almost always require the assistance of a programmer thoroughly familiar with the logic and conventions of the FIRM system.

An expanded treatment of the structure and operation of FIRM will appear in this journal in the near future and in the documentation distributed by CONDUIT.

\section{REFERENCES}

Eckerman, D. A. Developing a laboratory course using PASCAL on the Apple II. Behavior Research Methods \& Instrumentation, 1981, 13, 204-208.

Fischler, I. S. An on-line laboratory in cognition and perception. Behavior Research Methods \& Instrumentation, 1980, 12 116-119.

LEvy, C. M. Getting an on-line departmental teaching laboråtory on-line. Behavior Research Methods \& Instrumentation, 1980, 12, 111-113.

MaIN, D. B., \& HeAd, S. Computer simulations in the elementary psychological laboratory. Proceedings of the Conference on Computers in the Undergraduate Curricula. Hanover, N.H: Dartmouth College, June 1971.

Spelt, P. F. Bridging the gap between micros and minis in the teaching laboratory. Behavior Research Methods \& Instrumentation, 1981, 13, 198-200.

SPELT, P. F., \& WARden, J. A. WRIST-Wabash Research Investigation Simulation Teacher. Program library: PDP-11 Catalog. Maynard, Mass: DECUS, 1975.

Stout, R. L. Modeling and the Michigan Experimental Simulation Supervisor: An overview and some prospects. Behavior Research Methods \& Instrumentation, 1974, 6, 121-123.

Thurmond, J. B., \& Cromer, A. O. Models and modeling with the Louisville Experiment Simulation System (LESS). Behavior Research Methods \& Instrumentation, 1975, 7, 229-232.

White, K. D. An on-line laboratory in sensation and perception. Behavior Research Methods \& Instrumentation, 1980, 12 , 114-115. 